\title{
Impact of Band Rejection in Multichannel Broadband Subcarrier Multiplexing
}

\author{
Fernando A. Gutiérrez, Philip Perry, Frank Smyth, Andrew D. Ellis and Liam P. Barry
}

\begin{abstract}
This paper explores experimentally the impairments in performance that are generated when multiple single side band SCM signals are closely allocated in frequency to establish a spectrally efficient WDM link. The performance of cost effective single side band WDM/SCM implementations, without optical filters in the transmitter, presents a strong dependency on the imperfect sideband suppression ratio that can be directly achieved with the electro optical modulator. A direct detected broadband multichannel SCM link composed of a state of the art optical IQ modulator and five QPSK subcarriers per optical channel is presented, showing that a suppression ratio of $20 \mathrm{~dB}$ obtained directly with the modulator produced a penalty of $2 \mathrm{~dB}$ in overall performance, due to interference between adjacent optical channels.
\end{abstract}

Index Terms- Optical IQ modulator, sideband suppression ratio (SSR), subcarrier multiplexing (SCM), wavelength division multiplexing (WDM).

\section{INTRODUCTION}

$\mathrm{M}$ icrowave components present several advantages with respect to their optical counterparts like stability, low phase noise, frequency selectivity and reliable implementation of advanced modulation formats. Subcarrier multiplexing (SCM) leverages these properties to generate one or more digitally modulated radiofrequency (RF) signals that are then transmitted on an optical wavelength. Many SCM solutions have been reported addressing different applications like cable television (CATV) [1], radio over fibre [2], broadband point to point links [3], access networks [4] and $100 \mathrm{Gbit} / \mathrm{s}$ local area networks [5].

Multiple optical SCM channels can be combined in a wavelength division multiplexing (WDM) scheme $[6,7]$

Manuscript submitted on November 17, 2014; revised January 30, 2015. This work was supported in part by the EI CFTD grant CF/2011/1627, SFI grants 09/IN.1/12653 and 10/CE/I1853 and EPSRC grant EP/L000091/1.

F. A. Gutierrez, P. Perry, F. Smyth and L. Barry are with The RINCE Institute, Dublin City University, Glasnevin, Dublin 9, Ireland. (E-mail: fernando.gutierrez@dcu.ie).

F. Smyth is also with Pilot Photonics, Invent Centre, Dublin City University, Glasnevin, Dublin 9, Ireland.

A. D. Ellis is with Aston Institute of Photonic Technologies, Aston University, Aston Triangle, Birmingham B4 7ET, UK. increasing the capacity and the flexibility of the network. Usually, a single side band (SSB) implementation of each optical channel is performed (either with dual drive Mach Zehnder modulators (DD-MZM) [8] or with optical IQ modulators [9]) as a method to improve spectral efficiency and to eliminate dispersive fading [10]. In reality, the increased spectral efficiency obtained with a narrower allocation of optical channels will only be accompanied with optimum system performance when a high value of sideband suppression ratio (SSR) is ensured at the optical transmitter, as the remnants of the suppressed band will interfere with the subcarriers of the neighboring optical channel. SSR is limited by the imbalances and the extinction ratio of the optical modulators [11]. An SSR of 13 $\mathrm{dB}$ was achieved directly with a DD-MZM in [3]. Larger SSR values, up to $28 \mathrm{~dB}$, have been reported but using additional and costly optical filters [12, 13]. Fig.1 shows a spectrally efficient WDM/SSB transmitter without additional optical filters; where performance is limited due to the crosstalk associated with the imperfect suppression ratio.

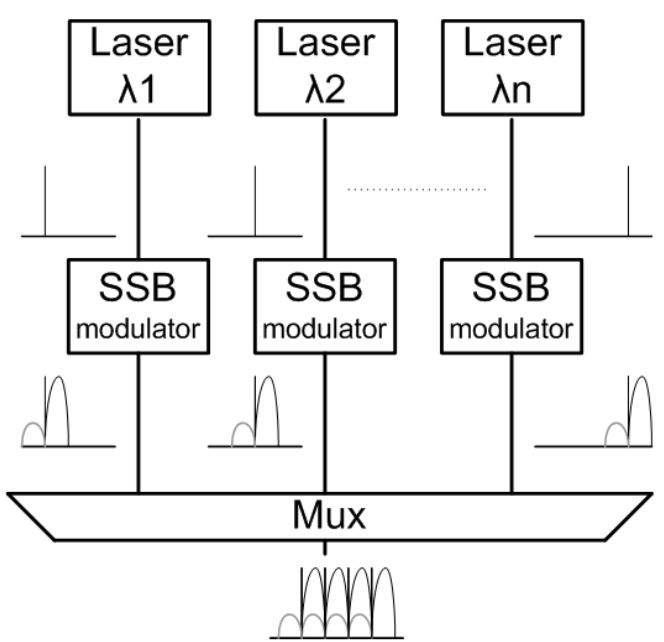

Fig. 1. WDM/SCM/SSB scheme. Each optical channel composed of one subcarrier. Crosstalk due to the imperfect SSB signals.

This paper investigates the design of a broadband direct detected WDM/SCM/SSB link with state of the art discrete optical IQ modulators and explores the incurred penalties in performance generated by the residual bands from the neighboring channel. An all-analogue real-time SCM/QPSK 


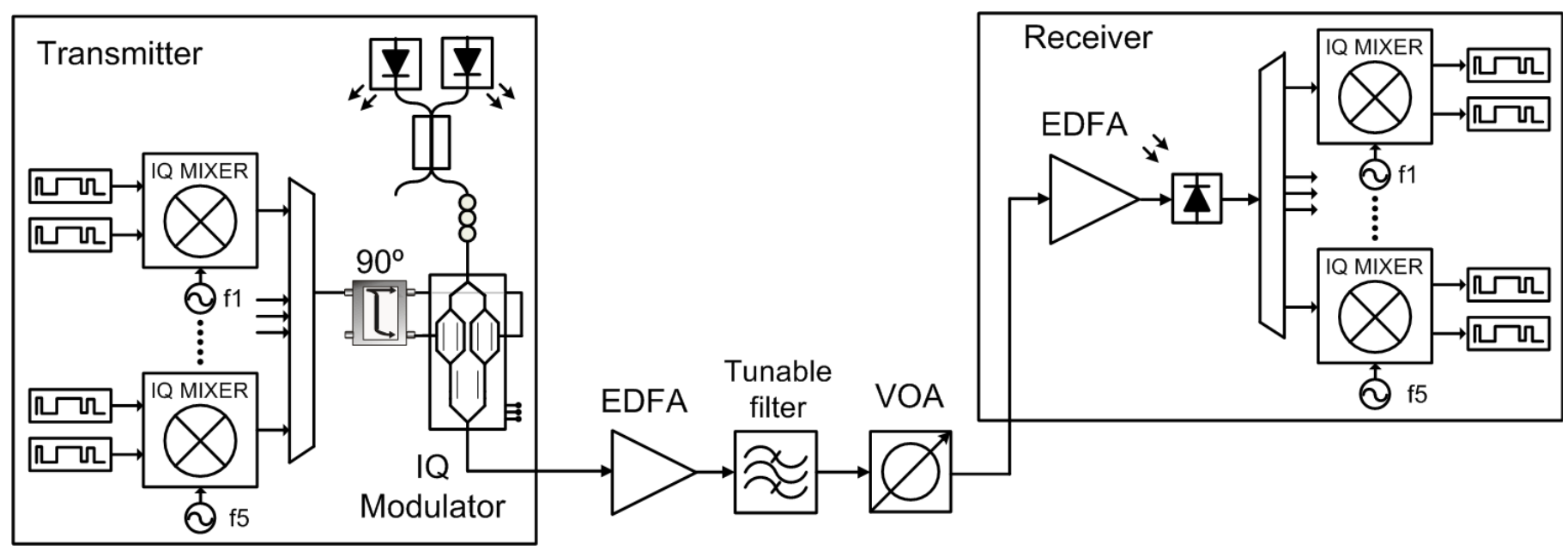

Fig. 2. Setup to emulate a WDM/SCM/SSB system. SSB generated with an optical IQ modulator. Each optical channel consists of 5 QPSK subcarriers. $2 \mathrm{~nm}$ band pass optical filters were used at the output of every EDFA but are omitted in the figure.

scheme has been designed for the experiments. Higher modulation orders would require demanding signal processing in the receiver [14] with the consequent penalty in the power consumption [15]. The presented all-analogue approach can offer a good trade-off between spectral efficiency and power consumption while using low-cost microwave components. Therefore, the implemented electrooptical transceiver would be suitable in metro/access networks or in radio over fibre systems, i.e. as an alternative for the increasingly important Common Public Radio Interface (CPRI) [16] in the upcoming 5G systems.

\section{MULTICHANNEL BROADBAND SCM/SSB SETUP}

The experiment was performed with the setup presented in Fig. 2. The RF transmitter consisted of five inexpensive off-the-shelf MMIC IQ mixers that were employed to upconvert ten uncorrelated bit streams that were previously filtered to reduce their spectrum to the first lobe of a sinc function. The performance of the RF IQ mixers was not optimized for spectrally dense broadband signals [17], so pseudo random binary sequences with a length limited to 127 bits were employed in these experiments. The nominal rate of every bit stream was $1.35 \mathrm{Gbit} / \mathrm{s}$ making a total rate of $13.5 \mathrm{Gbps}$ (2.7 Gbit/s on each $\mathrm{RF}$ carrier). The local oscillators (LO) were located at even harmonics of the data rate (from 5.4 to $16.2 \mathrm{GHz}$ ) such that there was no guard band between electrical subcarriers. After combining the five subcarriers a $90^{\circ}$ hybrid coupler was used to get the in phase and quadrature components required for the electrooptical IQ modulator to achieve SSB [9]. The RF receiver performed the splitting and demodulation of the electrical subchannels. For every RF band, the LO was generated and split to be used in the transmitter and the receiver, ensuring perfect frequency locking. Phase locking was accomplished with variable phase shifters in the receiver LO paths. The baseband signals at the output of the IQ mixers were fed to a bit error rate tester. No DSP techniques were employed at the transmitter or the receiver. It is worth noting that in a practical system, the electrical phase and frequency locking at the receiver could be achieved with an analogue PLL $[18,19]$.

The optical sources were two tunable lasers that allowed the adjustment of the separation between optical channels. The optical link was established with an optical IQ modulator plus an EDFA at the transmitter. The preamplified receiver consisted of an EDFA plus a $20 \mathrm{GHz}$ photo-detector. A tunable filter was used to select one of the optical channels.

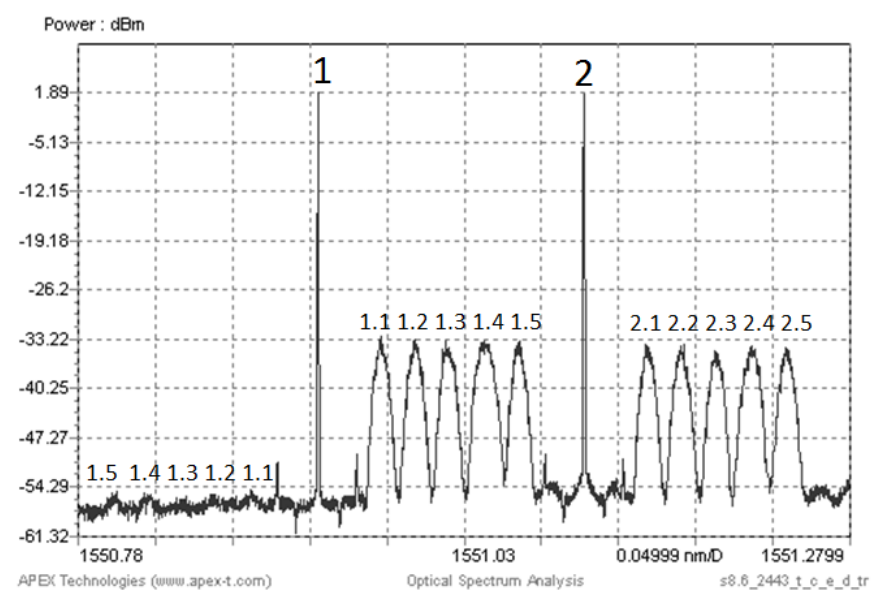

Fig. 3. Two optical SCM/SSB channels separated by $20 \mathrm{GHz}$. A sideband suppression ratio of more than $20 \mathrm{~dB}$ can be observed in channel 1.

The electro-optical conversion was performed with a state of the art thin film polymer optical IQ modulator [20], whose internal MZMs were biased at quadrature. This device presents the following properties: a bandwidth of 20 $\mathrm{GHz}$, small form factor, low $V_{\Pi}$ of 2.5 Volts and good bias point stability. These properties allow the design of a small low-power stable SCM/SSB transmitter that can achieve a good SSR without the need for additional optical filters in every channel. Fig. 3 shows an optical spectrum at the output of the EDFA at the transmitter, where two optical SCM/SSB channels are present and every channel and subchannel is numbered. On the optical channel 1 it can be seen that an SSR of more than $20 \mathrm{~dB}$ is achieved directly 
with the optical modulator. Simultaneously, that channel was experiencing interference from the sideband of the optical channel 2, which was not completely suppressed. Despite the fact that only one optical modulator was used in the experiment, as every subcarrier is interfered by the unsuppressed band of a different subcarrier, data and interference is still uncorrelated.

\section{MEASUREMENTS}

Firstly, the influence of the suppressed band on the performance of the neighboring channel was measured. The two optical channels were separated by $25 \mathrm{GHz}$ to operate on the ITU grid and to relax the selectivity of the optical filter at the receiver. The optical average power at the output of the transmitter EDFA was $5.5 \mathrm{dBm}$.

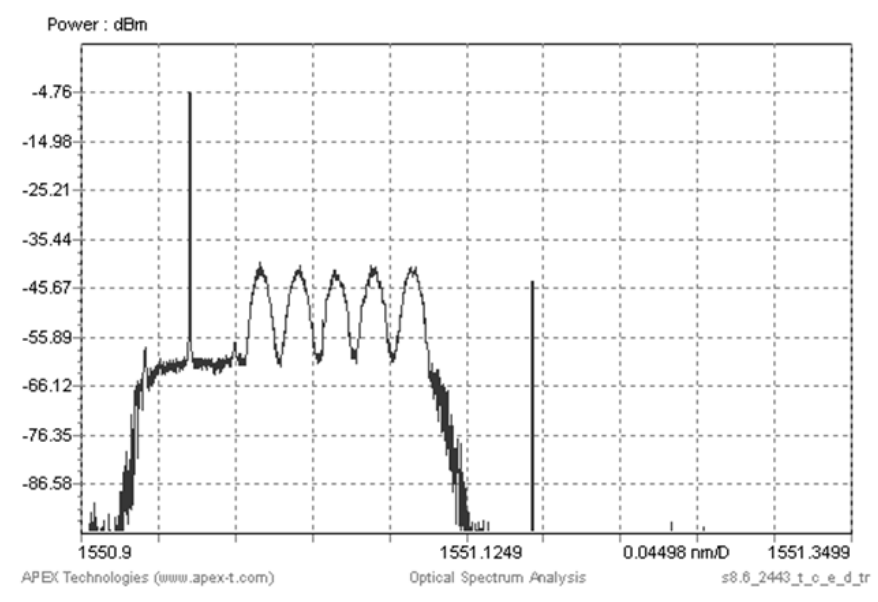

Fig. 4. Optical spectrum after filtering the two channel SCM/SSB signal in the receiver. The optical channels were separated 25 $\mathrm{GHz}$.

Overall performance was measured for optical channel 1 in two different situations: with and without the interference from the second optical channel. In the second case, instead of switching off the interfering channel, it was moved $100 \mathrm{GHz}$ away so that the gain of the EDFA at the transmitter remained the same for both channels. With this method it was ensured that the average optical power at the input of the pre-amplified receiver $\left(\mathrm{P}_{\text {IN }}\right)$ did not change in both situations and the measurements with and without interference could be compared. For the two optical channel transmission system, Fig. 4 shows the first channel after the optical filter in the receiver. The optical carrier of the interfering channel was reduced to $-40 \mathrm{dBc}$ with respect to the desired optical carrier. Measurements were focused on subchannels 1.1 and 1.4. With the $25 \mathrm{GHz}$ channel separation subchannel 1.1 was not interfered by any unsuppressed residual image and no penalty in performance was expected, in comparison to the case when the channel separation was $100 \mathrm{GHz}$, as confirmed by the bit error rate (BER) versus PIN curves in Fig. 5.

On the contrary, as it can be observed in Fig. 6, subchannel 1.4 was interfered by the remnants of the suppressed band in the neighboring channel with $25 \mathrm{GHz}$ channel separation. Considering a hard decision FEC with $7 \%$ overhead, the penalty due to this interference would be around $2 \mathrm{~dB}$ at the lowest sensitivity. Therefore, there is a tradeoff as spectral efficiency can only be maximized at the expense of reducing the maximum reach of the link in at least $10 \mathrm{~km}$. It should be noted that better overall receiver sensitivities could be achieved by applying carrier suppression in the transmitter. With the optical IQ modulator the optical carrier could be partially suppressed with the bias of the modulator without requiring any additional components, although at the expense of increased intermodulation distortion [21]. Relative performance differences between subchannel 1.1 and 1.4 were due to the behavior of the IQ mixers in different RF bands.

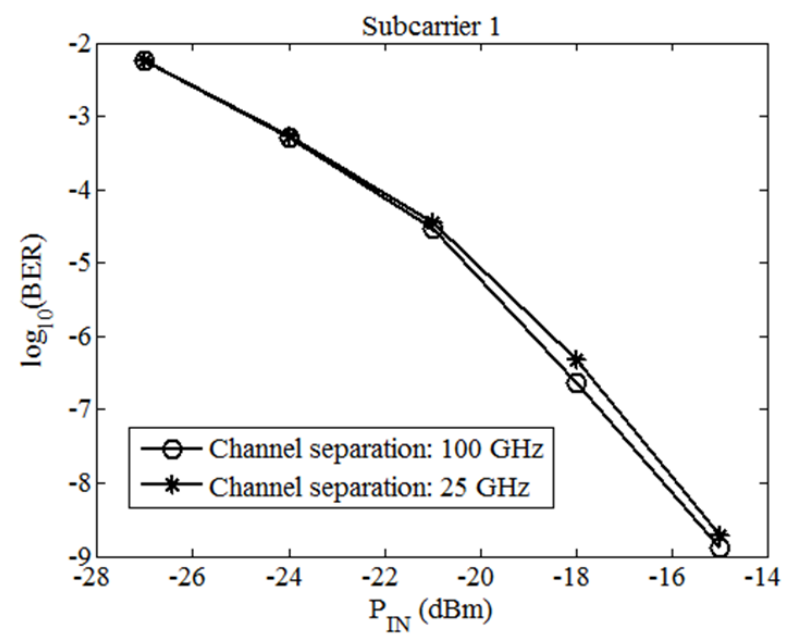

Fig. 5. Performance measured at subchannel 1.1 when the two transmitted optical channels are separated 25 and $100 \mathrm{GHz}$.

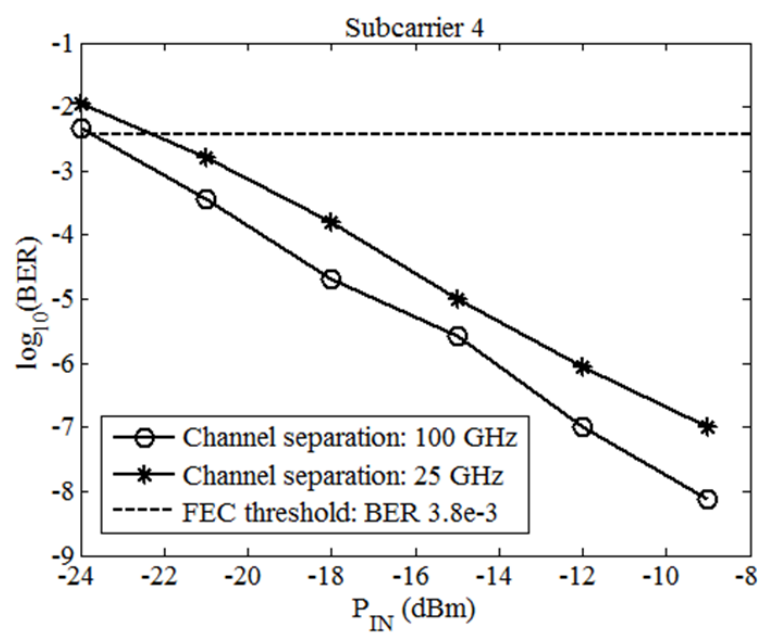

Fig. 6. Performance measured at subchannel 1.4 when the two transmitted optical channels are separated 25 and $100 \mathrm{GHz}$. Threshold for a 7\% hard decision FEC.

Another test was performed to study the influence of the frequency position of the unwanted sideband with respect to the desired signal. The optical channels were initially separated by $27.2 \mathrm{GHz}$ and this separation was increased in frequency steps of approximately $300 \mathrm{MHz}$, moving the location of the unsuppressed band that was interfering every subchannel. The maximum additional separation was $2.3 \mathrm{GHz}$, covering practically one sideband of an up- 
converted subchannel. The performance of subchannel 1.4 was measured maintaining a PIN of $-12 \mathrm{dBm}$ in all the cases. Fig. 7 illustrates the obtained BER measurements as a percentage with respect to the worst BER (9e-8). A minimum BER can be localized and corresponds to the situation in which the maximum peak of the interference from subchannels 2.4 and 2.5 coincides with the minimum level of the subchannel 1.4, as can also be observed in Fig. 7. The maximum values of BER are obtained in the two extremes of the picture, when maximum residual interference from subchannels 2.4 or 2.5 coincides approximately with the peak of subchannel 1.4. Both values of BER are similar showing that the swapping of the spectrum generated by subchannel 2.4 decorrelates the interference with subchannel 1.4 even when the original modulating data was the same.

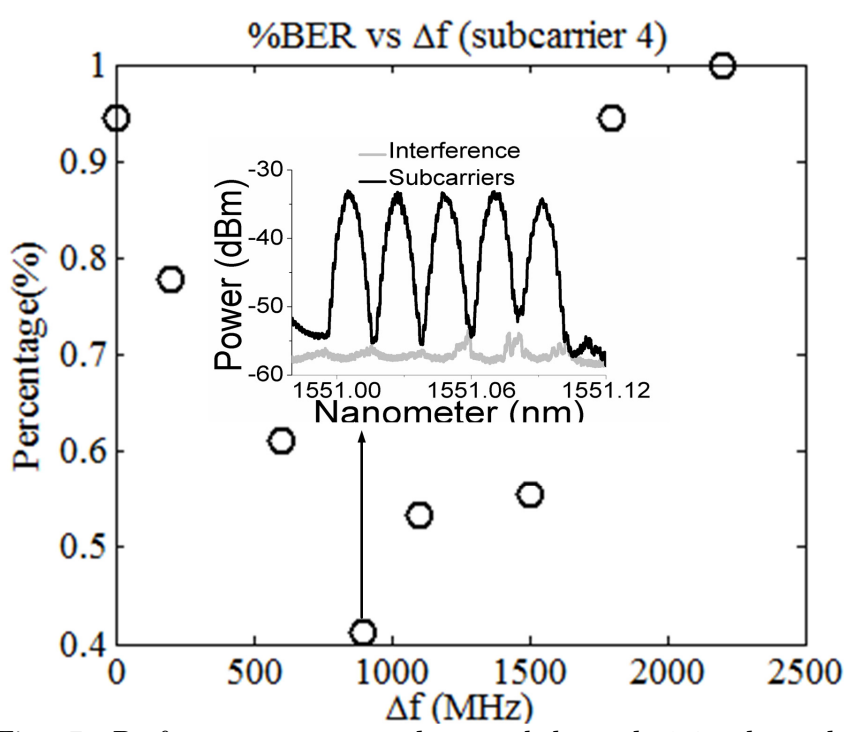

Fig. 7. Performance measured at subchannel 1.4 when the interfering optical channel is located at different frequencies. Inset shows the optical spectrum of subchannels and interference for the case of minimum BER.

Although other all-analogue broadband SCM/QPSK schemes have been reported [18, 22], the described setup and measurements are unique in several senses. Firstly, the use of MMIC IQ mixers demonstrates that a cost effective practical deployment with integrated discrete components is possible. Secondly, the employment of optical IQ modulators allows the implementation of colorless transmitters with partial optical carrier suppression without requiring optical filters in the transmitter. Finally, and despite the simplified transmitter, it has been proven that spectral efficiency is not compromised with a closer allocation of optical channels. These properties can motivate the application of this solution when flexibility and moderate transmission length are required but cost, power consumption, spectral efficiency and the future scalability determine the technology selected to be deployed. Metro/access networks or radio over fibre systems can leverage these features.

\section{Conclusions}

SCM is used in many applications employing analogue and digital implementations. The technique can be combined with WDM to increase the flexibility and the capacity of a network. To allocate closer optical channels, achieving improved spectral efficiency, optical SSB signals are required but the performance is limited by the sideband suppression ratio obtained at the electro-optic transmitter. This paper has presented a WDM/SCM/SSB link, based on a state of the art optical IQ modulator, where the penalty associated with the residual sideband from the neighboring optical channel was studied changing the separation between channels. A suppression ratio of more than $20 \mathrm{~dB}$ was achieved directly with the optical modulator without requiring additional optical filters and a penalty of less than $2 \mathrm{~dB}$ in overall performance of the WDM/SCM system was measured due to the associated interference. A fine adjustment of the frequency separation between optical channels can also be employed to ensure optimum performance in these systems, making the peaks of the residual interference coincide with the nulls of the desired subchannels.

\section{REFERENCES}

[1] P. M. Hill and R. Olshansky, "A 20-channel optical communication using subcarrier multiplexing for the transmission of digital video signals," J. Lightwave Technol., vol. 8, pp. 554-560, Apr. 1990

[2] G. H. Smith and D. Novak, "Broad-band millimeter-wave (38 $\mathrm{GHz}$ ) fiber-wireless transmission system using electrical and optical SSB modulation to overcome dispersion effects," IEEE Photon. Technol. Lett., vol. 10, pp. 141-143, Jan. 1998.

[3] R. Hui, B. Zhu, R. Huang, C. T. Allen, K. R. Demarest, and D. Richards, "Subcarrier multiplexing for high-speed optical transmission," J. Lightw. Technol., vol. 20, no. 3, pp. 417-427, Mar. 2002.

[4] B. Charbonnier, S. Menezo, P. O'Brien, A. Lebreton, J.M. Fedeli and B. Ben Bakir, "Silicon photonics for next generation FDM/FDMA PON," J. Opt. Commun. Netw., vol. 4, no. 9, pp. A29-A37, (2012).

[5] M. Salter, D. Platt, L. Petterson, L. Aspemyr, M. Bao, "Circuits and systems simulations for for $100 \mathrm{~Gb} / \mathrm{s}$ optical SCM transmission”, ICECS 2009, pp 960-963.

[6] M.S. Erkilinc, R. Maher, M. Paskov, S. Kilmurray, S. Pachnicke, H. Griesser, B.C. Thomsen, P. Bayvel and R.I. Killey, "Spectrally-Efficient Single-Sideband SubcarrierMultiplexed Quasi-Nyquist QPSK with Direct Detection”, in ECOC 2013, paper Tu.3.C.4.

[7] J. M. Buset, Z.A. El-Sahn and D.V. Plant, "Experimental Demonstration of a $10 \mathrm{~Gb} / \mathrm{s} 16-\mathrm{QAM}$ SCM WDM PON with Bandwidth-limited RSOA and IM/DD Transceivers," Proc. ECOC 2013, Tu.3.F.5.

[8] W. H. Chen and W. I. Way, "Multichannel single-sideband SCM/DWDM transmission systems," IEEE Journal of Lightwave Technology, vol. 22, no. 7, pp. 1679-1693, 2004.

[9] T. Nakatogawa, M. Maeda and K. Oyamada, "Optical single sideband modulator for distribution of digital broadcasting signals on millimetre-wave band based on self-heterodyne," Electron. Lett., vol. 40, no. 21, pp. 1369-1370, 14th October 2004.

[10] J. Maeda, T. Kato and S. Ebisawa, "Effect of fiber dispersion on subcarrier QAM signal in radio-over-fiber transmission," J. Lightwave Technol., vol. 30, no. 16, pp. 2625-2632, Aug. 2012.

[11] Y. Ogiso, Y. Tsuchiya, S. Shinada, S. Nakajima, T. Kawanishi, and H. Nakajima, "High Extinction-Ratio Integrated MachZehnder Modulator with Active Y-Branch for Optical SSB Signal Generation," IEEE Photon. Tech Lett. 22(12), 941-943 (2010). 
[12] T. Fujiwara and K. Kikushima, "140 Carrier, 20GHz SCM Signal Transmission across $200 \mathrm{~km}$ SMF by Two-step Sideband Suppression Scheme in Optical SSB Modulation," in Proc. OFC, 2007 paper OME2.

[13] S. J. Xiao and A. M. Weiner, "Optical carrier-suppressed single sideband (O-CS-SSB) modulation using a hyperfine blocking filter based on a virtually imaged phased-array (VIPA)," IEEE Photon. Technol. Lett., vol. 17, no. 7, pp. 1522-1524, Jul. 2005.

[14] L. Giorgi, F. Cavaliere, P. Ghiggino, F. Ponzini, A. Bianchi, and A. D'Errico, "Characterization of a high capacity multiuser optical access network using $1 \mathrm{~Gb} / \mathrm{s} 16$ QAM subcarrier multiplexing," J. Lightw. Technol., vol. 27, no. 9, pp. 12031211, May 1, 2009.

[15] A. Morea, S. Spandaro, O. Rival, J. Perello, F. Agraz, and D. Verchere, "Power management of optoelectronic interfaces for dynamic optical networks," ECOC, We.8.K.3, (2011).

[16] R. P. Almedia, R. S. Oliveira, N. S. Moritsuka, C. R. L. Frances, A. Teixeira, and J. C. W. A. Costa, "Digital Radio over Fibre transmission based on SCM and WDM system for C-Ran architecture" in Telecommunications Symposium (ITS), pp 1-5, Sao Paulo, 2014.

[17] B. E. Olsson, J. Mårtensson, A. Kristiansson, and A. Alping, "RF Assisted Optical Dual-Carrier 112 Gbit/s PolarizationMultiplexed 16-QAM Transmitter," Proc. OFC, San Diego, paper OMK5, 2010.

[18] P. Hill and R. Olshansky, "Bandwidth efficient transmission of $4 \mathrm{~Gb} / \mathrm{s}$ on two microwave QPSK subcarriers over a $48 \mathrm{~km}$ optical link," IEEE Photon. Technol. Lett., vol. 2, p. 510, (1990).

[19] P. Kourtessis and S.D. Walker, "A Complete 8-GHz QPSKMODEM Featuring Novel Subcarrier and Data Synchronization for Optical Communications", in IEEE Transactions on Communications, vol. 55, pp. 987-995, 2007.

[20] G. Yu, E. Miller, J. Mallari, C. Wei, B. Chen, H. Chen, V. Shofman and R. Dinu, "Small form factor thin film polymer modulators for telecom applications," in Proc. OFC, 2012, pp. 1-3, paper OM3J.

[21] F.A. Gutiérrez, P. Perry, Frank Smyth, A.D. Ellis, and L.P. Barry, "Optimum Bias Point in Broadband Subcarrier Multiplexing with Optical IQ Modulators" Journal Lightwave Technology (to be published).

[22] J. Y. Ha, A. Wonfor, R. V. Penty, I. H.White, and P. Ghiggino, "Spectrally efficient $10 \times 1 \mathrm{~Gb} / \mathrm{s}$ QPSK multi-user optical network architecture," presented at the OFC 2007, Anaheim, CA, OWD6. 